\title{
LOI RESTRICTIVE ET LOI PRODUCTIVE \\ DANS L'ÉTHIQUE FICHTÉENNE
}

\author{
Claude Piché, Université de Montréal
}

Ceci est une version de travail (« preprint »). Elle peut différer de la version publiée et ne doit pas servir pour fins de citation. La version finale est parue dans la Revue de métaphysique et de morale, juillet 2011, no. 3, p. 309-318.

The ENGLISH version of this text under the title "Fichte and the Universality of the Moral Law" can be found here on Papyrus.

RÉSUMÉ : Dans ses leçons de 1806 sur l'Initiation à la vie bienheureuse, Fichte établit une distinction entre la moralité supérieure et la moralité inférieure. Selon lui, l'éthique kantienne représente la forme paradigmatique de la moralité inférieure dans la mesure où elle commande l'uniformité de la conduite humaine en vertu de la loi morale. Or, bien que Fichte reconnaisse que son propre Système de l'éthique de 1798 comportait certains éléments de cette morale inférieure, il est possible de montrer que cet ouvrage évoquait déjà le rôle spécifique qu'est appelé à jouer chaque individu dans le monde. Ce qui constitue une anticipation du thème de la moralité supérieure.

MOTS CLÉS : Fichte, Kant, loi morale, morale inférieure/supérieure, uniformité

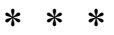

« Nous devons tous agir de la même manière. »Cette phrase percutante, et à première vue provocante, se trouve dans la cinquième section du $\S 18$ du Système de l'éthique, où Fichte traite du principe de la morale. Par cette affirmation à propos de l'« uniformité de l'agir » ${ }^{1}$, Fichte met en lumière le caractère universel de la loi morale. La question qui se pose alors est la suivante : que faut-il entendre par cette exigence d'uniformisation de l'agir de la part de Fichte ? Pour y répondre, il convient de porter attention à la manière dont ce dernier se démarque de Kant en ce qui a trait à l'interprétation de la loi morale. En effet, immédiatement après avoir énoncé sa

\footnotetext{
${ }^{1}$ System der Sittenlehre (ci-après SL), GA I, 5, 211, 213; tr. P. Naulin.
} 
thèse au $\S 18$, Fichte enchaîne avec un certain nombre de remarques, en partie critiques, à l'endroit de la conception kantienne de l'impératif catégorique.

Nous nous proposons donc dans ce qui suit de dégager la position de Fichte touchant l'universalisme en morale en établissant une comparaison avec Kant. Évidemment, Fichte s'est référé à la loi morale kantienne à plusieurs reprises au cours de sa carrière si bien que cette confrontation risquerait de nous amener très loin. Nous allons ici nous limiter aux précisions et aux réserves exprimées par Fichte dans le Système de l'éthique (1798) et à la critique qu'il adresse huit ans plus tard aux morales de type kantien dans l'Initiation à la vie bienheureuse. Dans ce dernier cas, la critique se fait plus radicale, au point où elle relègue la morale kantienne au statut de «morale inférieure ». Au terme de notre démarche, nous serons aussi conduits à nous interroger sur la cohérence de la lecture fichtéenne de la morale de Kant entre 1798 et 1806. En effet, la radicalité de la critique de 1806 en regard de celle que développe le Système de l'éthique est-elle l'indice d'une rupture dans l'itinéraire philosophique de Fichte, qui trouverait son point tournant dans l'accusation d'athéisme proférée contre lui en 1798-1799. C'est la question à laquelle nous tenterons de répondre à l'issue de notre parcours.

Dans le Système de l'éthique, Fichte est prêt à reconnaître le bien-fondé de la formulation générale de l'impératif catégorique selon Kant : « Agis de telle sorte que tu puisses penser la maxime de ton action comme principe d'une législation universelle »". Tel que formulé toutefois, ce commandement ne coïncide pas exactement d'après Fichte avec le principe moral lui-même, c'est-à-dire avec l'absolue autonomie (Selbstständigkeit) de la raison. Évidemment, cette indépendance et cette autonomie complètes de la raison doivent être reconnues par tous les êtres raisonnables et c'est pourquoi, dans sa formulation de l'impératif, Kant peut se permettre de faire appel à ceux-ci directement. À ce propos cependant, Fichte formule deux remarques. La

\footnotetext{
${ }^{2}$ SL, GA I, 5, 211.
} 
première souligne que l'appel à tous les êtres raisonnables lancé par Kant se situe au plan de l'« idée » et non de la réalité. Ainsi, en regard du caractère simplement idéal du consensus recherché, il convient de noter -- et Kant ne pourra qu'être d'accord -- que c'est de manière uniquement virtuelle que les autres individus raisonnables sont consultés dans cette procédure ${ }^{3}$. Celle-ci consiste pour ainsi dire en une expérience de pensée, en sorte que la réponse à la question posée à propos de l'universalité de la maxime ne peut venir en définitive que de l'individu raisonnable qui entame la procédure. Dans sa seconde remarque Fichte note que la formulation kantienne ne peut jouer au mieux qu'un rôle « heuristique » dans l'expérience morale. Elle vise à montrer que le recours à l'acceptabilité de la maxime à titre de loi n'est qu'un procédé visant à rassurer le sujet moral sur la valeur de sa maxime. Ainsi l'insistance sur l'universalité de la loi dans la formulation de l'impératif ne fait pas pour autant de cet impératif un principe « constitutif». Fichte tient en effet à replacer les choses dans leur juste perspective : ce n'est pas parce que la maxime est acceptable par tous qu'elle est morale, mais c'est au contraire parce qu'elle est en elle-même conforme à la raison qu'elle peut recevoir un accueil unanime. L'universalité de l'adhésion des individus raisonnables n'est en somme que la conséquence (Folgerung) de la rationalité de la maxime.

C'est bien sûr à partir de son propre « point de vue » que Fichte adresse à Kant ces deux remarque. Celles-ci peuvent dès lors nous servir de guide afin de cerner, par contraste, la position propre de Fichte en regard de la loi morale. Selon la seconde remarque, en effet, l'impératif kantien n'exprime pas le principe originaire, à savoir l'indépendance et l'autonomie de la raison en général. Or, comme la raison est ici considérée comme incarnée dans un individu, dans son «moi empirique », il faut que cet individu fasse abstraction de sa particularité et qu'il

\footnotetext{
${ }^{3}$ Dans ce texte à peu près contemporain du Système de l'éthique qu'est la Doctrine de la science nova methodo, Fichte s'interroge sur la légitimité du recours par Kant à l'assentiment d'autres êtres raisonnables, alors que ce dernier dans sa théorie omet de justifier la supposition même de l'existence de tels êtres. Cf. GA IV , 2, $141-142$.
} 
accède à la raison en général. Son jugement moral n'est valable que s'il s'élève à la raison. C'est dans la phrase qui au $\S 18$ précède immédiatement l'allusion à l'impératif kantien que Fichte énonce ce qui selon lui constitue véritablement le principe moral : «La fin morale dernière de chaque être raisonnable est, comme nous avons vu, l'autonomie de la raison en général, c'est-àdire la moralité de tout être raisonnable. Nous devons tous agir de la même manière $»^{4}$. Deux éléments retiennent l'attention dans cet extrait. En premier lieu, la thèse forte déjà évoquée selon laquelle tous les individus doivent agir de manière uniforme. La question est en fait de savoir ce qu'il faut entendre par cette uniformité : prend-elle place au plan du déroulement concret de l'action, ou alors uniquement au plan de la maxime? En second lieu, l'extrait indique que l'accès au principe moral se situe au sein de « chaque être raisonnable ». On l'aura noté, il n'y a pas ici, comme chez Kant, de référence à l'acceptabilité universelle. L'individu raisonnable, s'il sait s'élever à la raison en général, accède d'emblée au principe et sa démarche est de la sorte paradigmatique pour tout autre individu ${ }^{5}$. Or, les choses en restent-elles à ce niveau pour Fichte ? En vérité, cette approche monadique du principe premier de la morale n'est pas son dernier mot en cette matière. Elle intervient en fait au milieu d'une démarche dont la suite nous est annoncée cette fois dans la première remarque touchant l'impératif kantien, c'est-à-dire celle touchant le caractère simplement idéal de la consultation esquissée par Kant et que Fichte déplore. Dans la suite de cette section, Fichte s'applique précisément à montrer comment le consensus des individus raisonnables en matière de morale doit devenir concret dans les faits, comment il doit accéder à la réalité objective. Le consensus ne peut simplement rester au plan d'une expérience de pensée, d'un accord virtuel, à la manière kantienne. Pour Fichte, au contraire, c'est un devoir moral que de chercher à partager avec autrui sa conviction, si tant est qu'elle correspond vraiment

\footnotetext{
${ }^{4}$ SL, GA I, 5, 211.

${ }^{5}$ Voir à ce sujet Günter Zöller, « Die Individualität des Ich in Fichtes zweiter Jenaer Wissenschaftslehre (1796-99)», Revue Internationale de Philosophie, 206, 1998, p. 652-653.
} 
à ce que l'on pense. En effet, si elle est l'objet d'une adhésion sincère et profonde, alors on ne peut qu'être animé du désir de la partager, ou encore d'amener tous les autres individus à y souscrire. Nous ne voulons pas ici exposer en détail les étapes et les médiations proposées par Fichte pour réaliser de facto l'unanimité entre tous les individus moraux. Qu'il suffise de mentionner qu'au nombre de ces médiations il faut compter l'église et l'État. Mais c'est à la fin du $§ 18$ que Fichte indique l'instance sans doute la mieux à même de réaliser dans les faits ce consensus, encore qu'il s'agisse d'une tâche infinie : la « république savante $»^{6}$. Fichte, qui s'inspire dans ces pages largement du texte de Kant «Qu'est-ce que les Lumières ? », assimile en fait cette république à l'espace public évoqué par Kant, où tout un chacun, à condition qu'il sache lire et écrire, est invité à faire usage public de sa raison, afin d'y exposer et d'y mettre à l'épreuve ses convictions.

La fin du $\S 18$ nous éclaire sur deux choses. Elle permet d'abord de constater à quel point Fichte tient à dépasser la procédure kantienne confinée à la simple idéalité et à réaliser in concreto un consensus qui puisse à terme s'étendre à tous les êtres raisonnables. Par ailleurs, les considérations finales du paragraphe fournissent des indications sur la façon dont il faut comprendre l'« uniformité de l'action ». Contrairement à l'impression laissée par la phrase « nous devons tous agir de la même manière ", l'uniformité recherchée ici ne vise pas en définitive l'homogénéisation intégrale de l'agir humain, elle ne concerne pas le cours concret de l'action, au sens où tous les individus devraient poser exactement les mêmes gestes. La suite du paragraphe nous permet en effet de découvrir que l'uniformité se situe plutôt au plan des premiers principes, car c'est là seulement que l'unanimité, bien qu'en elle-même elle présente un défi redoutable, est recherchée : «Tous ont les mêmes convictions et la conviction de l'un est la conviction de

\footnotetext{
${ }^{6}$ SL, GA, I, 5, 224.
} 
tous...» ${ }^{7}$. On n'exige donc pas ici l'uniformité au plan de l'agir concret. L'accord sur les « premiers principes » est déjà une tâche suffisamment ardue. Seule la forme de la maxime dans l'expression « uniformité de l'agir » entre ici en considération, si bien qu'à cet égard Fichte ne se distingue pas foncièrement de Kant. Comme nous allons voir, le problème avec l'impératif catégorique se situe ailleurs et Fichte va montrer que la loi morale dans son acception kantienne demeure encore insatisfaisante dans la mesure où elle prône, à sa manière, une uniformité qui ne constitue qu'une étape préliminaire de l'agir moral authentique.

\section{L'Initiation à la vie bienheureuse publiée en 1806 est bien sûr un ouvrage consacré à la}

doctrine de la religion, mais on y trouve également, comme on sait, une très intéressante discussion sur la distinction entre morale inférieure et morale supérieure ${ }^{8}$. Afin de situer brièvement l'enjeu, qu'il nous soit permis ici de résumer le structure déployée par Fichte pour définir ce qu'il appelle les cinq différents points de vue possibles sur le monde. Ces cinq points de vue, Fichte les présente dans leur ordre hiérarchique, de sorte que cela ne préjuge en rien de l'ordre de leur occurrence dans l'histoire. Chacune de ces visions du monde est en effet susceptible d'intervenir à un moment ou à un autre dans le cours du temps. Ces points de vues décrivent en fait ce qui dans chaque cas est envisagé par l'homme comme étant la « réalité »du

${ }_{8}^{7} S L$, GA I, 5, 226; cf. 221.

${ }^{8}$ Il va de soi que, d'un point de vue purement théorique, le strict équivalent du Système de l'éthique de 1798 se trouve dans les leçons sur l'éthique de 1812. On note toutefois que Fichte dans ces leçons n'a pas recours à la distinction entre la moralité supérieure et la moralité inférieure. Il paraît se soucier davantage de dresser un tableau cohérent de son éthique, sans tenter de confiner Kant au niveau inférieur de la moralité, comme il le fait dans son texte de 1806 sur la Vie Bienheureuse. En fait, même si Fichte formule en 1812 quelques remarques critiques à l'endroit de la conception kantienne de la moralité, son appréciation de la philosophie morale de Kant est dans l'ensemble élogieuse. Ceci nous incite à penser que Fichte dans son écrit populaire sur l'Initiation à la vie bienheureuse est moins intéressé à présenter sa philosophie morale de manière précise et nuancée qu'à procéder à l'exposition claire et simplifiée des deux point de vue à partir desquels l'éthique peut être envisagée. Ceci étant admis, il n'est pas interdit de faire appel à la distinction entre moralité supérieure et moralité inférieure énoncée en 1806 afin d'évaluer les passages de la Sittenlehre de 1798 déjà examinés eu égard à l'articulation de l'individualité et de l'uniformité de l'agir. En d'autres termes, le recours à la dichotomie établie par Fichte entre les deux niveaux de moralité est autorisé - et s'avère en fait hautement révélateur - pour autant que nous gardions à l'esprit qu'elle est conçue pour être comprise d'un plus large public et qu'elle a par conséquent, pour reprendre l'expression de Max Weber, le statut d'une distinction entre deux « types idéaux ». 
monde. Le premier point de vue est celui du monde sensible envisagé comme réalité ultime. Le second est celui de la « loi de l'ordre et du droit identique » pour tous et représente la moralité inférieure. Le troisième est celui de la moralité supérieure et il situe la réalité dans le bien, la beauté et le sacré. En quatrième lieu intervient la religion, qui considère ces trois sphères comme procédant en vérité de Dieu, alors que le cinquième point de vue est celui de la science, de la science au sens le plus noble, c'est-à-dire la philosophie 9 .

À l'évidence, c'est à partir du troisième point de vue, celui de la morale supérieure, que Fichte formule sa critique de la loi morale kantienne, laquelle relève du second point de vue, celui de l'ordre et du droit appliqué à tous. La morale kantienne est en fait présentée ici comme emblématique de ce point de vue, qui aux yeux de Fichte apparaît nettement insuffisant ${ }^{10}$. Or, ce qu'il y a de remarquable ici, c'est que Fichte n'hésite pas à associer au sein du second point de vue loi juridique et loi morale. On sait en effet combien Fichte a toujours insisté sur la nette séparation entre ces deux sphères. Mais, dans le cas présent, c'est ce que ces deux sphères ont en commun qui retient son attention. Le fait que la morale de Kant se présente sous la forme d'une loi laisse entrevoir qu'elle partage avec le droit certains traits ${ }^{11}$. Ainsi le texte évoque-t-il de façon générale la loi qui introduit un ordre et qui, pour ce faire, possède une fonction, si l'on ose dire, égalisatrice : Fichte emploie les participes présents ordnend et gleichend. Dans ce dernier cas, la loi morale, tout comme la loi juridique, uniformise : nous sommes tous égaux devant la loi, également soumis à la loi. Par ailleurs, la loi juridique prescrit, mais le plus souvent sous forme d'interdiction, si bien qu'elle proscrit certaines actions plus qu'elle n'en génère. Or ces traits propres à la loi juridique sont aussi à leur manière présents dans la loi morale kantienne et

\footnotetext{
${ }^{9}$ Anweisung zum seligen Leben (ci-après Anweisung), GA I, 9, 106-112; cf. WL 1804-II, GA II, 8, $416,418$.

${ }^{10}$ Du moins ce jugement est-il valable pour la philosophie morale de Kant jusqu'à la Critique de la raison pratique. Cf. Anweisung, GA I, 9, 108. Dans sa lettre à Jacobi du 8 mai 1806, Fichte avoue en revanche qu'il se sent assez proche du Kant de la Critique de la faculté de juger, point culminant de la spéculation kantienne : GA III, 5, 356.

${ }^{11}$ Fait à signaler, Schleiermacher critiquait à la même époque la morale fondée sur l'« impératif catégorique » en la qualifiant de « juridique ». Voir Brouillon zur Ethik (1805/06), Hambourg, Meiner, 1981, p. 47, cf. p. 4.
} 
ils font peser sur celle-ci une lourde hypothèque, en sorte qu'aux yeux de Fichte elle se manifeste, elle aussi, et malgré son statut distinct par rapport au droit, sur un mode essentiellement négatif. Et ceci est vrai à deux niveaux : au plan de la procédure tout autant qu'au plan de la vie morale en tant que telle.

Si la loi morale est appelée à servir de critère pour établir la valeur d'une action, ou mieux de la maxime qui y préside, force nous est d'avouer que ce critère fonctionne d'abord et avant tout par exclusion. Ainsi, par exemple, la procédure mise en place par Kant visant à vérifier la possible universalisation de la maxime aboutit presque invariablement à des interdictions. C'est à tout le moins ce que l'on peut conclure de la mise en œuvre de cette procédure dans la Fondation de la métaphysique des mours. En effet, les exemples examinés consistent tous en des maximes à prohiber : le suicide, la fausse promesse etc. ${ }^{12}$ Dans chacun de ces cas, le test se solde par un échec et c'est à ce titre que l'impératif catégorique kantien sert de critère pour la vie morale. Il vise d'abord à écarter la maxime inacceptable et c'est précisément cet aspect négatif que constate Fichte dans l'extrait suivant. Après avoir évoqué la doctrine du droit, Fichte affirme qu'il en va de même de l'éthique habituelle « qui veille uniquement à ce que personne ne fasse tort à autrui et à ce que chacun s'abstienne de tout ce qui est contraire au devoir, soit qu'il y ait ou qu'il n'y ait pas de loi juridique expresse qui le défende ${ }^{13}$. L'impératif catégorique s'avère donc n'être au total qu'un critère négatif pour la vie morale. Son rôle consiste à interdire et à prohiber, si bien que par son aspect restrictif il introduit à sa manière une uniformité, celle de tous les sujets moraux en tant qu'ils s'abstiennent de mal agir.

Mais alors, c'est toute la vie morale de l'individu qui revêt un aspect négatif. En effet, la loi morale exige le renoncement en regard de l'égoïsme et de l'amour de soi. C'est sans doute

\footnotetext{
${ }^{12}$ Kant, Grundlegung zur Metaphysik der Sitten, AK IV, 421-423.

${ }^{13}$ Anweisung, GA I, 9, 107-108, tr. (modifiée) M. Bouiller. Cf. Über das Wesen des Gelehrten (1805), GA I, 8, 77.
} 
elle qui fonde la dignité de l'être moral, mais cette dignité se manifeste comme une victoire sur les inclinations, comme un renoncement à la primauté de la jouissance offerte par le monde sensible. Le second point de vue donc, celui de la moralité inférieure, se définit comme un détachement délibéré face au premier point de vue, celui de la réalité du monde sensible. Le mérite moral de l'individu réside dès lors dans sa force de résistance face aux inclinations et aux désirs. De la sorte, la loi de la moralité, tout comme la loi juridique, est un facteur d'ordre, elle introduit « équilibre et repos », là où régnaient le chaos et la discorde. Par là, l'individu moral fait à coup sûr preuve de liberté, mais cette liberté se définit essentiellement comme Selbstständigkeit, qu'il convient ici de traduire par indépendance tout autant que par autonomie. L'essence de l'être raisonnable fini tient à cette autosuffisance, en sorte que l'« unique fondement, l'unique preuve de l'indépendance de l'homme dans ce système, c'est la loi morale se révélant dans son for intérieur » ${ }^{14}$. La loi confère ainsi à l'individu sa « réalité », mais la liberté qu'elle dévoile ne peut être interprétée que de manière négative, que comme une totale autarcie face aux sollicitations du monde sensible. L'individu agit exclusivement par respect pour la loi. En conséquence, nous avons affaire à une liberté et à une autonomie purement « formelles » qui ne témoignent pas d'une attitude positive face au monde, mais plutôt de ce que Fichte désigne comme l'« apathie » qui caractérise la morale stoïcienne. Lorsqu'elle est comprise de manière conséquente, la moralité inférieure ne vise qu'une chose : faire en sorte qu'à aucun moment on ne puisse apparaître indigne à ses propres yeux et par là éprouver du mépris pour soi (Selbstverachtung). Pour le reste, d'après Fichte, ce second point de vue culmine dans une totale indifférence et il donne lieu à une morale vide de contenu, dépourvue de toute orientation positive ${ }^{15}$.

\footnotetext{
${ }^{14}$ Anweisung, GA I, 9, 107.

${ }^{15}$ Anweisung, GA I, 9, 148, 149.
} 
Évidemment, c'est à partir de la moralité supérieure, qu'il qualifie aussi de moralité « authentique $»^{16}$, que Fichte est en mesure de critiquer le second point de vue. Le concept de loi y est certes maintenu, mais cette fois dans une acception clairement positive. Cette loi vient étendre sa juridiction précisément sur le territoire laissé vacant par le second point de vue : l'action morale concrète dans le monde. Or, si l'on nous permet de revenir un instant à la terminologie juridique, cette loi de la moralité supérieure ne doit en aucun cas être interprétée comme une lex permissiva, au sens où, en l'occurrence, elle désignerait l'espace laissé libre par ce qui n'est pas encadré par la loi juridique. Au contraire, il s'agit d'une loi productive, d'une loi sui generis ayant pour fin de façonner concrètement le monde sensible pour y faire apparaître le monde intelligible.

La question qui se pose dès lors est la suivante : comment s'articule cette loi productive avec la loi morale restrictive du second point de vue. En tout état de cause, la loi kantienne de l'autonomie formelle n'est pas carrément reniée par Fichte. Elle constitue pour ainsi dire un passage obligé et pour cette raison elle demeure incontournable ${ }^{17}$. Son rôle, nous l'avons vu, consiste à introduire ordre et équilibre dans le monde social et dans le monde intérieur. Mais la moralité supérieure permet de mettre en perspective cette loi d'allure somme toute juridique afin de faire voir que l'idéal héroïque mais stérile du stoïcien ne peut représenter une fin en soi, encore moins le dernier mot en matière de morale. Il s'agit d'une étape nécessaire mais uniquement préparatoire, qui demande à être dépassée.

Qu'en est-il maintenant de la teneur de cette loi de la moralité authentique ? Fichte, pour la décrire, utilise le mot erschaffend, qui signifie produire, mais aussi créer. La loi de la morale supérieure est donc une loi de création qui régit les gestes de l'individu dans son action concrète.

\footnotetext{
${ }^{16}$ Anweisung, GA I, 9, 154.

${ }^{17}$ Anweisung, GA I, 9, 109.
} 
Or, celui-ci a-t-il une conscience claire de la règle qu'il met en œuvre au moment où il agit de la sorte ? Rien n'est moins sûr. Fichte fait référence à cet égard au « talent », qui est un don naturel, voire un don divin, et qui pour se déployer ne requiert nullement, comme il le souligne, l'injonction d'un impératif catégorique. À ce niveau, la loi n'est pas ressentie comme une obligation contraignante. Par là, Fichte tient à mettre en valeur la dimension de spontanéité et d'absence de contrainte formelle qui caractérise l'action novatrice. Ce n'est d'ailleurs pas un hasard s'il a recours au concept de génie pour décrire l'activité au plan de la moralité supérieure $^{18}$. Il est bien connu, à tout le moins depuis Kant, que le génie procède à partir de règles, qui demeurent toutefois hors de portée de l'artiste lui-même. Il ne faut donc pas s'attendre à voir Fichte faire état du contenu de ces règles de l'action créatrice. Qu'il suffise de souligner que selon lui le génie n'est pas uniquement le fait de l'artiste, mais qu'il a une portée plus large, incluant par exemple l'homme d'État et le philosophe. Il est du reste d'autant plus difficile de définir en général la teneur de la loi productrice que celle-ci trace pour chaque individu un itinéraire propre à l'intérieur d'un projet d'ensemble, qui n'est rien de moins que la manifestation de l'être absolu, de l'être suprasensible. En accédant à la moralité supérieure, le sujet moral se voit partie prenante d'un dessein global dans lequel un rôle particulier lui est dévolu, qui ne revient qu'à lui et au service duquel il est appelé à consacrer toutes ses énergies : « Chacun, dis-je, sans exception reçoit une part de l'être suprasensible qui lui est exclusivement propre et qui n'appartient à aucun autre individu hors de lui...» ${ }^{19}$. En se mettant ainsi au service de la phénoménalisation de l'être, le sujet moral est appelé à devenir en toute connaissance de cause l'« instrument» de cette manifestation du suprasensible dans le sensible. Et pour ce il est amené à abandonner sa « volonté propre » au profit du plan d'ensemble. Si le second niveau de la

\footnotetext{
${ }^{18}$ Anweisung, GA I, 9, 157,161. Cf. Über das Wesen des Gelehrten (1805), GA I, 8, 81.

${ }^{19}$ Anweisung, GA I, 9, 160.
} 
vision du monde impliquait le renoncement au monde sensible, alors le troisième niveau implique à son tour que l'individu renonce à l'autoaffirmation et transcende l'autonomie formelle fondée sur la loi morale kantienne. En fait, c'est de plein gré que le sujet autonome (selbstständig), lorsqu'il accède à la morale supérieure, renonce à l'affirmation de soi comme entité morale distincte. Or, ce qu'il y a de remarquable dans ce passage librement consenti, c'est qu'en abandonnant les prétentions de sa personnalité morale propre, le sujet moral accède à vrai dire à un principe d'individuation plus spécifique encore qui situe de manière précise son rôle unique et non substituable à l'intérieur d'un monde conçu comme totalité organique. En d'autres mots, si la loi morale kantienne garantissait l'autonomie formelle et la dignité intrinsèque de l'être raisonnable, la loi créatrice, en faisant de l'individu un instrument au service de ce que Fichte considère être la cause la plus noble - la manifestation de l'être absolu --, lui confère une dignité supérieure à la première et le fait accéder à la « liberté accomplie $»^{20}$.

Ces quelques remarques sur la conception fichtéenne du troisième niveau de vision du monde nous auront entre autres permis de faire apparaître les spécificités de même que les limites d'une morale qui, comme celle de Kant, est axée sur une loi formelle telle qu'on la trouve à l'œuvre au second niveau, celui de la moralité inférieure. Il convient pour terminer d'aborder brièvement la question de la cohérence même de l'œuvre de Fichte, puisque ce dernier avoue clairement dans l'Initiation que son Système de l'éthique de la période d'Iéna s'inscrit sans contredit et de plain-pied dans la seconde vision du monde ${ }^{21}$. Faut-il voir ici une évolution, une rupture ? Dans son article sur la théorie fichtéenne de l'individualité éthique, Edith Düsing se penche sur cette question et cherche à évaluer les conséquences qu'a entraînées cette (prétendue)

\footnotetext{
${ }^{20}$ Anweisung, GA I, 9, 161. Reinhard Lauth a pour sa part tenté d'élaborer une réponse kantienne à la caractérisation par Fichte de la morale de Kant comme une morale de l'interdiction et de la prohibition. À cet effet, il mobilise les devoirs dits « larges » ou encore « imparfaits» dont il est question dans la Fondation. Voir son article « Elementare Pflicht und höhere Moral », dans le même, Vernünftige Durchdringung der Wirklichkeit. Fichte und sein Umkreis, Neuried, ars una, 1994, p. 407-419.

${ }^{21}$ Anweisung, GA I, 9, 108.
} 
césure dans l'itinéraire de Fichte qu'est l'Atheismusstreit. Or, elle parvient à retracer une continuité au sein de l'œuvre en montrant que le Fichte de la période tardive n'écarte pas tout simplement les développements du Système de l'éthique. Il est au contraire en mesure de maintenir une articulation essentielle entre « autonomie », préoccupation centrale pour la période d'Iéna, et « théonomie », thème amplement développé dans la période plus tardive ${ }^{22}$. Pour notre part, nous tenons à souligner qu'une relecture attentive du Système de l'éthique à la lumière des développements de 1806 permet d'y découvrir plusieurs indices de ce qui deviendra la vision du monde propre à la moralité supérieure.

\footnotetext{
${ }^{22}$ Edith Düsing, « Autonomie - soziale Heteronomie - Theonomie. Fichtes Theorie sittlicher Individualität », Fichte-Studien, 8, 1995, p. 74-75. Cf. Marco Ivaldo, « „Das Wort wird Fleisch“. Sittliche Inkarnation in Fichtes später Sittenlehre », dans H. G. von Manz et G. Zöller (dir.), Fichtes praktische Philosophie. Eine systematische Einführung, Hildesheim, Olms, 2006, p. 190.
} 